\title{
La captura y almacenamiento de carbono: una novedosa técnica de reducción de emisiones de gases de efecto invernadero regulada por la Unión Europea
}

\section{Carbon capture and storage: a novel technique for reducing greenhouse gas emissions regulated by the European Union}

\author{
ÍNIIGO SANZ RUBIALES*
}

Resumen: El presente artículo desarrolla las principales aristas de la captura y almacenamiento del dióxido de carbono emanado de las grandes instalaciones de combustión, novedosa técnica que al tornarse imprescindible para la reducción de emisión de gases de efecto invernadero ha sido regulada por la Unión Europea (UE) a través de la directiva 2009/31. Así, tras reconocer los aspectos jurídicos relevantes de su regulación —obligatoriedad, permisos de exploración y almacenamiento, entre otros - se incide en el régimen de responsabilidad y garantías.

Palabras clave: captura y almacenamiento del dióxido de carbono - gases de efecto invernadero - permisos de exploración y almacenamiento responsabilidad ambiental - responsabilidad por cambio climático transferencia de responsabilidad

Summary: This article deals with main aspects of a novel technique for carbon dioxide capture and storage released by large combustion plants. Since this novel technique has become essential for reducing greenhouse gas emissions, it has been regulated by the European Union through the Directive number 2009/31. Therefore, acknowledging relevant legal aspects for regulation, such as: mandatory, exploration permits, storage among others, the focus has been pointed out on responsibilities and guarantees regime.

Key words: carbon dioxide capture and storage - greenhouse gases exploration permits and storage - environmental liability - climate change liability - liability transfer

CONTENIDO: I. LA CAPTURA Y ALMACENAMIENTO DE CARBONO Y SU IMPLANTACIÓN EN LA UNIÓN EUROPEA.- I.1. UNATECNOLOGÍA INNOVADORA.I.2. UNA TÉCNICA IMPRESCINDIBLE PARA UNA REDUCCIÓN AMBICIOSA DE EMISIONES DE GASES DE EFECTO INVERNADERO.- I.3. LA RÁPIDA ADOPCIÓN DE UNA NORMATIVA REGULADORA Y SUS RAZONES.- I.4. APLICABILIDAD DE LA TECNOLOGÍA DE CAPTURA Y ALMACENAMIENTO DE CARBONO A LAS DENOMINADAS "ECONOMÍAS EMERGENTES».- II. ASPECTOS JURÍDICOS

* Catedrático acreditado de Derecho Administrativo en la Universidad de Valladolid. Correo electrónico: isanz@der.uva.es 
RELEVANTES DE LA REGULACIÓN.-- II.1. LA CAPTURA Y ALMACENAMIENTO DE CARBONONOESOBLIGATORIATODAVÍA PARALASGRANDESINSTALACIONES EMISORAS.- II.2. CONTROL PREVIO DEL ALMACENAMIENTO DE $\mathrm{CO}_{2}$ EN EL SUBSUELO: LOS «PERMISOS» DE EXPLORACIÓN Y ALMACENAMIENTO.II.3. EL CARÁCTER MARGINAL DE LA CAPTURA Y EL TRANSPORTE DE $\mathrm{CO}_{2}$ EN LA REGULACIÓN.- II.3.1. CAPTURA: LIMITACIONES AMBIENTALES.II.3.2. TRANSPORTE: EL ACCESO DE TERCEROS A LA RED.- III. TÉCNICAS ECONÓMICAS. EL PRINCIPIO «QUIEN CONTAMINA, PAGA»: RESPONSABILIDAD Y RÉGIMEN DE GARANTÍAS.- III.1. RESPONSABILIDAD AMBIENTAL.III.2. "RESPONSABILIDAD POR DAÑOS CLIMÁTICOS».- III.3. GARANTÍAS FINANCIERASYTRANSFERENCIA DERESPONSABILIDAD.-IV.RECAPITULACIÓN.

\section{LA CAPTURA Y ALMACENAMIENTO DE CARBONO Y SU IMPLANTACIÓN EN LA UNIÓN EUROPEA}

\section{I.1. Una tecnología innovadora}

La CAC (captura y almacenamiento de carbono) es una técnica que consiste en capturar el dióxido de carbono (el más abundante gas de efecto invernadero) emitido por una determinada instalación industrial, transportarlo e inyectarlo en un emplazamiento subterráneo dentro de una formación geológica adecuada con vistas a su almacenamiento permanente. No se trata únicamente de almacenar el gas bajo la tierra, en galerías, o en oquedades subterráneas. Se trata de que mediante complejos procesos tecnológicos, físicos y químicos el $\mathrm{CO}_{2}$ quede atrapado en estructuras subterráneas indefinidamente $(\mathrm{o}$, al menos, durante cientos o miles de años) e incluso pueda desaparecer al transformarse y mineralizarse en la estructura rocosa en la que sea inyectado.

El proceso de almacenamiento industrial del carbono comprende tres fases: captura, transporte y almacenamiento ${ }^{2}$.

Captura. Son muy diversas las fuentes emisoras de $\mathrm{CO}_{2}$. En la medida en que solo se pueden capturar — con la tecnología actual— los gases procedentes de fuentes fijas, los principales emisores proceden de la industria y de las centrales térmicas de producción de electricidad. Las tecnologías para la captura son muy variadas. La captura puede realizarse antes, durante o después de la combustión. Las de tecnologías de postcombustión son las más adaptable para las centrales térmicas actuales y la captura puede realizarse a través de diversas técnicas. En

1 Alenza García, José Francisco. «El nuevo régimen legal del almacenamiento geológico de dióxido de carbono". Revista de Administración Pública, 185 (2011), pp. 289-322, p. 291.

2 Ibídem, p. 292. 
todo caso, se trata de separar el dióxido de carbono del resto de los gases producidos por la instalación emisora.

Transporte. Como el almacenamiento se debe llevar a cabo en lugares especialmente aptos para ello, lo normal será que deba realizarse un transporte del carbono desde el lugar de captura al emplazamiento de almacenamiento. Antes del transporte, el carbono debe ser comprimido para reducir su volumen y que llegue al emplazamiento de almacenamiento con la presión necesaria para su inyección. Normalmente se utilizan tuberías ad hoc o gasoductos, y empiezan ya a ser calificados como «ceoductos», pero se puede hacer también por buques o camiones cisterna.

Almacenamiento. Se puede llevar a cabo en diversos tipos de almacén subterráneo. Los principales son los yacimientos agotados de gas o petróleo, las formaciones salinas profundas y las capas de carbón profundas o no explotables. La inyección se realiza sobre rocas porosas que hacen de almacén, a más de ochocientos metros de profundidad (porque a partir de esa profundidad la presión permite almacenar el gas en volúmenes mucho menores que los requeridos en superficie).

Lógicamente, en el estado actual de la ciencia, el coste de estas instalaciones solo se compensa con fuertes estímulos, un precio del carbono relativamente elevado y un volumen importante de dióxido de carbono almacenado (no emitido).

\section{I.2. Una técnica imprescindible para una reducción ambiciosa de emisiones de gases de efecto invernadero} No es posible alcanzar una economía "descarbonizada» únicamente mediante la imposición del mercado de emisiones y, en su caso, de impuestos específicos: es preciso también hacer «rupturas tecnológicas» ${ }^{3}$. Pero, como señala el Informe Stern sobre la economía del cambio climático, incluso en el caso de que se potencien más aun las energías renovables (sobre todo, eólica y solar) o las tecnologías bajas en carbón, los combustibles fósiles seguirán representando más del cincuenta por ciento del suministro energético global para el $2050^{4}$, por lo que la tecnología CAC, conocida habitualmente por sus siglas en inglés como CCS (carbon capture and storage) se configura como imprescindible para alcanzar los ambiciosos objetivos de reducción de emisiones del Protocolo de Kioto 5 .

3 DE MARLIAVE, Luc. «Le captage et le stockage géologique de $\mathrm{CO}_{2}$ ". En FALQUE, Max \& Henri LAMOTTE (eds.). Changement climatique et pollution de l'air. Droits de propriété, Économie et Environnement. Bruselas: Bruylant, 2010, p. 347.

4 Stern Review On The Economics of Climate Change (http://webarchive.nationalarchives.gov.uk/+/ http://www.hm-treasury.gov.uk/independent_reviews/stern_review_economics_climate_change/ stern_review_report.cfm), 2008, pp. 222, 234.

5 Comunicación de la Comisión sobre Producción sostenible de electricidad a partir de combustibles fósiles. Conseguir centrales eléctricas de carbón con emisiones próximas a cero después de 2020.

LA CAPTURA Y

ALMACENAMIENTO

DE CARBONO:

UNA NOVEDOSA

TÉCNICA DE

REDUCCIÓN

DE EMISIONES DE

GASES DE EFECTO

INVERNADERO

REGULADA

POR LA UNIÓN

EUROPEA

CARBON CAPTURE

AND STORAGE:

A NOVEL

TECHNIQUE

FOR REDUCING

GREENHOUSE

GAS EMISSIONS

REGULATED BY

THE EUROPEAN

UNION 
Lógicamente, si la utilización de la tecnología CAC resulta imprescindible a nivel global, esa necesidad se agudiza en el caso de la Unión Europea (UE), que ha asumido, convencionalmente y, después, unilateralmente, unos compromisos de reducción de emisiones mucho más ambiciosos que el resto de los Estados parte del Protocolo de Kioto. Aunque Europa camina hacia una progresiva implantación y expansión de tecnologías de producción de energía renovable, no pueden eliminarse de golpe las centrales térmicas (que generan electricidad a partir de combustibles fósiles) por varios motivos $^{6}$; a) por una parte, razones de abastecimiento estratégico, con referencia, en especial, a la utilización de carbón europeo: una de las finalidades de la potenciación de las energías renovables en el ámbito de la UE es la reducción de la dependencia exterior, especialmente respecto de países políticamente inestables, que podrían colapsar la economía europea mediante la adopción de decisiones políticamente desacertadas; b) por otra parte, motivos económicos: muchas de las centrales térmicas hoy en funcionamiento todavía no se han amortizado, y debería dárseles unos plazos suficientemente amplios para lograr recuperar las inversiones realizadas, haciéndolo compatible — en la medida de lo posible - con la adopción de medidas para la reducción de emisiones de gases de efecto invernadero en el territorio europeo; c) pero, además, como señala el Informe Stern, la utilización apropiada de técnicas de captura y almacenamiento del carbono permitiría mantener la utilización de combustibles fósiles y evitaría una excesiva caída de los precios de los combustibles sólidos, y ello sin daño para la atmósfera ${ }^{7}$; d) finalmente, hay también razones sociales: el mantenimiento del empleo de los millares de trabajadores que trabajan en el sector minero en diversos países europeos.

Los análisis de la Comisión señalan que en 2030 la captura y almacenamiento de carbono podrían eliminar el noventa por ciento de las emisiones de las centrales térmicas generadoras de electricidad y contribuir a reducir un veinte y cinco las emisiones globales de la UE en relación con las del año $2000^{8}$.

En todo caso, al regular los proyectos CAC, la Unión Europea ha señalado con claridad que esta tecnología se encuentra entre las

Bruselas, 10/01/2007, COM(2006) 843 final, p. 6. Si la tecnología CAC se aplica correctamente, el almacenamiento evita muy eficazmente las emisiones a la atmósfera: como señalaba el Informe especial del Grupo Intergubernamental de Expertos sobre el Cambio Climático (IPCC) sobre «La captación y el almacenamiento del dióxido de carbono» (2006), con base en la experiencia existente se puede afirmar que la fracción de $\mathrm{CO}_{2}$ retenida en depósitos geológicos debidamente seleccionados y gestionados posiblemente exceda del noventa y nueve por ciento en cien años.

6 SANZ RUBIALES, Íñigo. «El almacenamiento geológico de dióxido de carbono en la directiva 2009/31, de 23 de abril». Revista Aranzadi de Derecho Ambiental, 19 (2011), pp. 75ss.

7 Stern Review On The Economics of Climate Change, pp. 186-187, 222.

8 Producción sostenible de electricidad a partir de combustibles fósiles. Conseguir centrales eléctricas de carbón con emisiones próximas a cero después de 2020. Ob.cit., p. 16. 
opciones técnicas utilizables para reducir las emisiones de carbono en el territorio comunitario.

\section{I.3. La rápida adopción de una normativa reguladora y sus razones}

En muy pocos años la Unión Europea tradujo los resultados de diversos estudios sobre las ventajas de una técnica hasta el momento casi desconocida - la captura y almacenamiento de carbono- en una directiva que ha sido transpuesta ya por los Estados miembros.

En efecto, solo recientemente se alude a esta tecnología en los documentos de la Unión Europea. En concreto, el Plan Estratégico Europeo de Tecnología Energética (EETE), aprobado en 2007, incluyó, junto a las energías renovables y otras tecnologías de baja emisión de carbono, la técnica de captura y almacenamiento de carbono9. Tras los análisis y proyecciones de futuro realizados por las autoridades europeas, la Unión decidió incluir en el paquete «energía y cambio climático» la elaboración de una directiva sobre el almacenamiento geológico de dióxido de carbono que vio la luz en abril de 2009 (la directiva $2009 / 31)^{10}$, y que fue transpuesta por España mediante la ley 40/2010, de 29 de diciembre, Ley de Almacenamiento Geológico de Dióxido de Carbono, aprobada con un gran consenso y tras una tramitación parlamentaria en la que se plantearon escasas enmiendas (al margen de cuestiones competenciales) ${ }^{11}$. Se trata de una ley peculiar: muy técnica y muy pegada a la directiva ${ }^{12}$, general en la concepción y en la redacción, pero «casi singular» en la práctica porque no es previsible que se ejecute un número elevado de proyectos CAC. Ahora bien, son necesarios: España necesita aplicar la tecnología CAC porque no puede prescindir, a medio plazo, de la energía térmica ${ }^{13}$.

Esta premura por regular la técnica CAC llama más la atención aun si se tiene en cuenta que la utilización de esta tecnología lleva aparejados riesgos en buena medida desconocidos actualmente. Pero esta rapidez tiene su razón — sus razones— de ser: son varias las causas que han

9 Comunicación de la Comisión de 22 de noviembre de 2007. Plan Estratégico Europeo de Tecnología Energética (Plan EETE) - Hacia un futuro con baja emisión de carbono. COM(2007) 723 final.

10 Directiva 2009/31/CE del Parlamento Europeo y del Consejo de 23 de abril de 2009 relativa al almacenamiento geológico de dióxido de carbono y por la que se modifican la directiva 85/337/ CEE del Consejo, las Directivas 2000/60/CE, 2001/80/CE, 2004/35/CE, 2006/12/CE, 2008/1/CE y el Reglamento (CE) no 1013/2006 del Parlamento Europeo y del Consejo (DOUE de 6 de mayo de 2009).

11 AlenZa García, José Francisco. Ob. cit., pp. 289, 299.

12 lbídem, pp. 299, 302, 303-304ss..

13 Álvarez Pelegry, Eloy. «La captura y almacenamiento de $\mathrm{CO}_{2}$ : una solución eficiente para luchar contra el cambio climático». Real Instituto Elcano, (http://www.realinstitutoelcano.org/wps/ portal/rielcano/contenido?WCM_GLOBAL_CONTEXT=/elcano/elcano_es/zonas_es/dt20-2010), 2010: «Puede parecer una contradicción, [pero] es clara la necesidad del ahorro y la eficiencia y las renovables, pero los ciclos combinados y el carbón son necesarios para facilitar la necesaria energía rodante al sistema y dar firmeza a las renovables».

\section{LA CAPTURA Y ALMACENAMIENTO DE CARBONO: \\ UNA NOVEDOSA \\ TÉCNICA DE \\ REDUCCIÓN}

DE EMISIONES DE

GASES DE EFECTO

INVERNADERO

REGULADA

POR LA UNIÓN

EUROPEA

CARBON CAPTURE

AND STORAGE:

A NOVEL

TECHNIQUE

FOR REDUCING

GREENHOUSE

GAS EMISSIONS

REGULATED BY

THE EUROPEAN

UNION 
llevado al legislador comunitario a adoptar esta regulación con tanta diligencia ${ }^{14}$. Estas son, en síntesis, las siguientes: en primer lugar, una razón de seguridad jurídica para los eventuales inversores en los procesos de investigación y de comercialización. A pesar de las incertidumbres vinculadas con la aplicación de esta tecnología, no parece factible profundizar en el costoso desarrollo tecnológico de este mecanismo sin «un marco regulatorio estable que asegure quién puede inyectar, dónde, cómo y con qué consecuencias en caso de fugas o daños al medio ambiente» ${ }^{15}$. La seguridad de la captura, transporte y almacenamiento de $\mathrm{CO}_{2}$ requiere una regulación adecuada.

Por otra parte, los Estados, además de regulación, pueden establecer estímulos (fundamentalmente económicos) para potenciar la inversión privada en este tipo de instalaciones ${ }^{16}$. No debe olvidarse que, actualmente, la captura y almacenamiento de dióxido de carbono son extraordinariamente caros: la tecnología CAC actual tiene un coste netamente superior al del mercado de cuotas que permitiría compensarlo ${ }^{17}$. De ahí la necesidad de un marco de estímulo que permita potenciar el uso asequible de la tecnología CAC.

La relativa urgencia tiene también su razón de ser en que se trata - la CAC — de una tecnología de transición, cuya aplicación no se plantea a largo plazo, sino a corto y, sobre todo, medio plazo, en el ínterin del proceso de sustitución de fuentes energéticas basadas en los combustibles fósiles por fuentes renovables. En efecto, la propia propuesta de directiva de la Comisión la califica como técnica «de transición» (considerando 4); y lo es porque tiene una utilidad meramente coyuntural, mientras prosigue el desarrollo y la implantación de energías alternativas (renovables o también «fósiles sostenibles») a la quema de combustibles fósiles tradicionales en la producción de electricidad, pero no pretende reducir en ningún caso el apoyo a las políticas de ahorro energético, de fomento de energías renovables y de estímulo de tecnologías de bajo consumo de carbono (considerando 4 de la directiva) ${ }^{18}$.

14 GÁmEZ MEJÍAS, Manuel. «El marco jurídico del almacenamiento geológico profundo de dióxido de carbono en el contexto de la lucha contra el cambio climático. Perspectivas y reflexiones tras la transposición al derecho interno». Revista Aranzadi de Derecho Ambiental, 20 (2011), pp. 49-79, pp. 51-52.

15 Ahora bien, como se verá mas abajo, la directiva tiene mucho de experimental y provisional, a la vista del escaso desarrollo de la tecnología CAC. Por eso la regulación tiene una escasa densidad normativa, por la falta de datos suficientes que permitan prever razonablemente cómo van a funcionar estas instalaciones. Por eso, para compensar esta «falta de densidad normativa» la directiva obliga a la introducción de planes estatales que reduzcan la incertidumbre o, al menos, la canalicen mediante procedimientos administrativos que garanticen la participación social, al amparo del artículo 4 de la directiva (decisión sobre las zonas para almacenar, etcétera), (ibídem, p. 62).

16 Ver International Energy Agency. CO2 Capture and Storage. A key carbon abatement option. París: OECD, 2008, p. 124.

17 En principio, algunas empresas han calculado unos costes de entre setenta y cien euros por tonelada de $\mathrm{CO}_{2}$ captada y almacenada; esto exige, necesariamente, reducir costes mediante nuevos procedimientos (DE MARLIAVE, Luc. Ob. cit., p. 349).

18 «La captura y el almacenamiento geológico de carbono (CAC) es una tecnología de transición que contribuirá a mitigar el cambio climático [...]. Esta tecnología no debe ser un incentivo para aumentar 
Además, este marco regulador de la captura, transporte y almacenamiento de dióxido de carbono ya ha sido adoptado por algunos otros países desarrollados, tanto para poner en funcionamiento plantas de demostración, como para la explotación comercial (por ejemplo, Australia, algunas provincias canadienses ${ }^{19}$, etcétera) y la UE no quiere perder en este ámbito concreto el liderazgo que ha venido asumiendo en la lucha contra el cambio climático global.

\subsection{Aplicabilidad de la tecnología de captura y almace- namiento de carbono a las denominadas «econo- mías emergentes»}

La tecnología CAC no solo es importante para que la UE alcance sus objetivos de reducción de emisiones, sino que también puede resultar especialmente eficaz en la reducción de emisiones de las economías emergentes cuyo crecimiento se basa en modelos energéticos vinculados a la quema de combustibles fósiles ${ }^{20}$. En los últimos años se ha dado algunos pasos, todavía vacilantes, en esa dirección. En 2005, laUEy China celebraron un acuerdo de cooperación sobre captura y almacenamiento de carbono dentro del «Plan de Acción sobre tecnologías limpias del carbón», complementado por la posterior cumbre UE-China, del mismo año, que incluía también un compromiso político para desarrollar y demostrar en China y en la UE la tecnología CAC antes de $2020^{21}$.

A la Unión Europea le preocupan de forma especial la evolución exponencialmente creciente de las emisiones de gases de efecto invernadero (GEI) en las economías emergentes dependientes del carbón. Según las proyecciones del Grupo Intergubernamental de Expertos sobre el Cambio Climático (IPCC) se espera que, en ausencia de medidas adicionales, las emisiones de $\mathrm{CO}_{2}$ procedentes del uso de

la proporción de las centrales eléctricas que utilizan combustibles fósiles. Su desarrollo no debe llevar a la reducción de los esfuerzos de apoyo a las políticas de ahorro energético, a las fuentes renovables de energía y a otras tecnologías seguras y sostenibles con baja emisión de carbono, ni en el ámbito de la investigación ni en el financiero". Esta consideración busca tranquilizar a aquellos interlocutores de la Comisión que durante el procedimiento de elaboración de la propuesta de directiva mostraron su preocupación por el riesgo de desviación de los esfuerzos destinados a la eficacia energética y a las energías renovables, como señala la Exposición de Motivos de la Propuesta de directiva del Parlamento Europeo y del Consejo relativa al almacenamiento geológico de dióxido de carbono y por la que se modifican las Directivas del Consejo 85/337 y 96/61, y las Directivas 2006/60/CE, 2001/80/CE, 2004/35/CE, 2006/12/CE y el Reglamento (CE) no 1013/2006, $\operatorname{COM}(2008) 18$ final, Bruselas, 23 de enero de 2008, n. 2.

19 Fundamentalmente Alberta y British Columbia. Ver, al respecto, KRUPA, Henry J. «Legal Framework for Carbon Capture and Storage in Canada». En AUTORES VARIOS. Carbon Capture and Storage. Emerging Legal and Regulatory Issues. Oxford: Hart Publishing, 2011, pp. 39ss. Ver igualmente, THOMSON, Drew \& Anshuman KHARE. «Carbon Capture and Storage (CCS) Deployment - Can Canada Capitalize on Experience?». Journal of Technology, Management \& Innovation, III, 4 (2008), pp. 111ss.

20 Stern Review On The Economics of Climate Change, p. 222.

21 Con anterioridad, en 2004, se firmó un acuerdo bilateral preliminar de colaboración en materia de energía entre China y EE.UU. que debería desarrollarse, en buena medida, mediante la cooperación en proyectos de captura y almacenamiento de carbono (GIDDENS, Anthony. La política del cambio climático. Madrid: Alianza Editorial, 2010, p. 258). 
la energía se incrementen en el período 2000-2030 entre un cuarenta y cinco y un ciento diez por ciento. De dos tercios a tres cuartos de ese incremento procederán de las regiones en desarrollo. Aun tomando medidas incisivas en materia de energías renovables y otras tecnologías de baja emisión de carbono, se estima que los combustibles fósiles proporcionarán en 2050 la mitad del abastecimiento mundial de energía, tal y como reconoce la Comunicación de la Comisión sobre Demostración de la captura y el almacenamiento geológico del carbono $(\mathrm{CAC})$ en los países en desarrollo emergentes: financiación del proyecto UE-China de central de carbón con emisiones próximas a cero, de 25 de junio de 2009 (COM(2009) 284 final).

En efecto, la Comisión aporta en esta Comunicación algunos datos sobre la utilización de combustibles fósiles en China que hacen tambalear la creencia en la eficacia de las medidas unilaterales europeas. En este país el carbón está en el origen del setenta por ciento de la energía producida. Y las perspectivas de futuro a corto y medio plazo no hacen mas que confirmar que el desarrollo del país oriental se basa en una economía del carbón: a título de ejemplo, «solo en 2007, China construyó el equivalente de una central eléctrica de carbón de 500 MW cada dos días y medio. Esto representa un incremento en las emisiones de China de alrededor de $4 \mathrm{Mt}$ de $\mathrm{CO}_{2}$ por semana solo en la generación de electricidad a partir del carbón» ${ }^{22}$.

Ante estos datos, hay que recordar que la Unión Europea solo produce un diez por ciento de las emisiones de GEI mundiales, por lo que las ambiciosas medidas planteadas por la política comunitaria de lucha contra el cambio climático pueden quedar en agua de borrajas si la comunidad internacional (en especial, las economías emergentes) no se suma, en mayor o menor medida, a esta iniciativa. La UE corre el peligro de avanzar sola: lidera la carrera contra el cambio climático, pero para liderar tiene que haber seguidores, si se quiere evitar un esfuerzo aparentemente inútil... Por eso no debe faltar la ambición, pero tampoco el realismo, porque la Unión Europea es solo una pequeña — comparativamente cada vez más pequeña— parte del mundo ${ }^{23}$.

Además, no lo olvidemos, los costes de implantación de la tecnología CAC supondrán necesariamente una reducción de la competitividad de las empresas europeas frente a las instalaciones ubicadas en las economías

22 Sobre las emisiones de $\mathrm{CO}_{2}$ en China, su proyección a medio plazo y el papel del carbón en la producción energética, ver ZHAO, Qiuyan. "China's Emerging Regulatory Framework for Safe and Effective CCS, 2011-15». En AUTORES VARIOS. Carbon Capture and Storage. Emerging Legal and Regulatory Issues, pp. 223ss. El vigente plan quinquenal 2011-2015 incluye ya la tecnología CAC como una prioridad. Empiezan a ser ya numerosos los proyectos de demostración de esta tecnología en aquel país, ver p. 227.

23 Ver SANZ RUBIALES, Íñigo. «Notas sobre la hoja de ruta hacia una economía hipocarbónica competitiva en 2050 (Comunicación de la Comisión de 8 de marzo de 2011, COM(2011) 112 final)». Revista Catalana de Dret Ambiental, II, 1 (2011), p. 17. 
emergentes. En la medida en que no son obligatorias las instalaciones CAC en la UE, de acuerdo con la directiva, su generalización en Europa exigiría el dictado de normas obligatorias (por ejemplo, de estándares de emisión, como veremos más adelante) o establecer regímenes de ayudas ad hoc; el coste estimado es de diez mil millones de euros anuales. Esto, a su vez, generará previsiblemente problemas de competitividad. Y «si los principales competidores de la UE no se comprometen del mismo modo, la UE tendrá que considerar la manera de reducir aun más el riesgo de fuga de carbono derivado de esos costes suplementarios» ${ }^{24}$.

\section{ASPECTOS JURÍDICOS RELEVANTES DE LA REGULACIÓN}

\section{II.1. La captura y almacenamiento de carbono no es obligatoria todavía para las grandes instalaciones emisoras}

Durante el proceso previo a la presentación de la propuesta de directiva, la Comisión introdujo, en el «proyecto de marco reglamentario» que presentó en una reunión a gran escala con los principales interlocutores (el 8 de mayo de 2007), una disposición que establecía la CAC como obligatoria a partir de una cierta fecha. Como recuerda la propia Comisión en la Exposición de Motivos de la propuesta definitiva, la obligatoriedad de la tecnología CAC fue bien recibida por algunos de los consultados (principalmente organizaciones ambientalistas) y cuestionada por otros, que argüían que la tecnología no estaba lo suficientemente desarrollada como para imponerla obligatoriamente y que dicha imposición podía tener consecuencias impredecibles. Como consecuencia de esta oposición, la Comisión evaluó las posibles implicaciones económicas, sociales y medioambientales y decidió posponer sine die la obligatoriedad de la técnica ${ }^{25}$.

Por lo tanto, los actuales titulares de las instalaciones industriales emisoras de $\mathrm{CO}_{2}$ no están obligados a implantar estos mecanismos (para los futuros tampoco se ha fijado un dies a quo de exigencia de implantación). Únicamente están previstas las obligaciones de evaluación y de las reservas de espacio para las correspondientes instalaciones que la directiva exige a aquellos grandes emisores que obtengan la primera licencia de construcción regulada en la directiva 2001/80, relativa a la limitación de emisiones a la atmósfera de determinados agentes contaminantes procedentes de grandes instalaciones de combustión, con posterioridad a la

LA CAPTURAY

ALMACENAMIENTO

DE CARBONO:

UNA NOVEDOSA

TÉCNICA DE

REDUCCIÓN

DE EMISIONES DE

GASES DE EFECTO

INVERNADERO

REGULADA

POR LA UNIÓN

EUROPEA

CARBON CAPTURE

AND STORAGE:

A NOVEL

TECHNIQUE

FOR REDUCING

GREENHOUSE

GAS EMISSIONS

REGULATED BY

THE EUROPEAN

UNION 
entrada en vigor de la directiva 2009/31 (artículo 33 de esta última). Es decir, únicamente deberán asegurar que las nuevas instalaciones están preparadas para que se les añadan posteriormente tecnologías de captura y almacenamiento del $\mathrm{CO}_{2}$ (capture ready) ${ }^{26}$.

Ahora bien, si pretenden llevar a cabo dicha actividad CAC deberán someterse a la regulación de esta directiva 2009/31. Y por supuesto, en un futuro próximo (2015), la tecnología se podría imponer obligatoriamente a los grandes emisores, si se demuestra su viabilidad económica y ambiental, de acuerdo con la propia directiva.

\subsection{Control previo del almacenamiento de $\mathrm{CO}_{2}$ en el subsuelo: los «permisos» de exploración y de almacenamiento}

La directiva no prejuzga el régimen jurídico del subsuelo en los diversos Estados miembros, a efectos de la regulación de los emplazamientos para el almacenamiento de carbono ${ }^{27}$. Sin embargo, resulta evidente que la calificación jurídica del subsuelo es determinante del régimen jurídico de obtención de los permisos de investigación y almacenamiento que debe establecer cada Estado, en desarrollo de la directiva.

Como ya dejamos señalado en su momento ${ }^{28}$, el nombre utilizado por la directiva, «permiso», tiene connotaciones «autorizatorias». Sin embargo, lo cierto es que dicho «permiso» previsto por la directiva puede plasmarse en instrumentos jurídicos estatales diferentes, en función del ordenamiento jurídico aplicable en cada Estado miembro. Así, lo que la directiva califica como «permiso de exploración» está dirigido a estudiar los posibles emplazamientos de los almacenes subterráneos ${ }^{29}$ y se transpone igualmente en la ley española como «permiso de investigación» — similar a los permisos de investigación mineros-. Según el artículo 5.2 de la directiva, estos permisos deben concederse o denegarse en procedimientos abiertos a todas las entidades con capacidad suficiente y «sobre la base de criterios objetivos, publicados y no discriminatorios». Y según la ley española tienen naturaleza autorizatoria o incluso «preconcesional»: otorgan a su titular prioridad para obtener la concesión, pero no es imprescindible para ello ${ }^{30}$.

26 Ver CHIAVARI, Joana. «The legal Framework for Carbon Capture and Storage in the EU (Directive 2009/31/EC)". En Oberthür, Sebastian \& Marc Pallemarts (eds.). The New Climate Policies of the European Union. Bruselas: Brussels University Press, 2010, pp. 161-162, 167.

27 En la línea de la tradicional previsión del Derecho comunitario, hoy reconocida en el artículo 345 del Tratado de Funcionamiento de la Unión Europea (TFUE): «Los Tratados no prejuzgan en modo alguno el régimen de la propiedad en los Estados miembros».

28 SANZ RUBIALES, íñigo. «El almacenamiento geológico de dióxido de carbono en la directiva 2009/31, de 23 de abril», p. 80.

29 Según la directiva (artículo 3.9), el «permiso de exploración» es «la decisión escrita y razonada por la que se autoriza la exploración y se especifican las condiciones en las que debe realizarse, expedida por la autoridad competente de conformidad con las disposiciones de la presente directiva».

30 AlenZA García, José Francisco. Ob. cit., pp. 311-313. 
Sin embargo, los permisos de almacenamiento ya no responden, en la legislación española, a meras «autorizaciones» ${ }^{31}$. La demanialización de los almacenes subterráneos que realiza la ley española provoca que la utilización exclusiva de dichos espacios a efectos de almacén de dióxido de carbono deba venir habilitada por una concesión demanial, y no por una mera autorización (a pesar de que en la Ley de Minas bastaba con este último título para la utilización en exclusiva de estructuras subterráneas: artículo 34). A priori, la posición del solicitante ante una concesión y una autorización es netamente diferente: recuérdese que las concesiones son constitutivas del derecho concedido, mientras que las autorizaciones son, en principio, meramente declarativas del derecho preexistente en el patrimonio del peticionario, sin perjuicio de la existencia de otras diferencias ${ }^{32}$. Por eso, la habilitación para utilizar una formación geológica apta para ello como almacén de dióxido de carbono viene dada, en el ordenamiento jurídico español, por una «concesión de almacenamiento», tal y como lo prevé la ley (artículos 4.11 y 10).

Hay que entender, en definitiva, que "permiso» es, en la directiva, un instrumento jurídico-administrativo cuya obtención se configura como conditio sine qua non para el ejercicio válido de la actividad. La preexistencia o no del derecho a su obtención, la mayor o menor libertad del ejercicio de esa actividad importa poco. Aquí la soberanía del Estado se reconoce sin ambages por la propia directiva (artículo 4.1), que recuerda que el Estado tiene «derecho» (debería decir «potestad») a permitir o no el establecimiento de almacenes subterráneos de $\mathrm{CO}_{2} \mathrm{y}$ a determinar, en su caso, la ubicación de estos:

Los Estados miembros mantienen el derecho de decidir las zonas en las que podrán situarse los emplazamientos de almacenamiento, de conformidad con los requisitos de la presente directiva. Este derecho incluye el derecho de los Estados miembros a no permitir almacenamiento alguno en partes de su territorio o en la totalidad del mismo ${ }^{33}$.

En lo que se refiere a los depósitos subterráneos de $\mathrm{CO}_{2}$, la discrecionalidad estatal opera ex ante (puede o no permitir en su territorio la existencia de este tipo de estructuras); pero si admite dicha posibilidad, la configuración legal del procedimiento se convierte en una actuación reglada, más próxima en la práctica a las autorizaciones que a las concesiones ${ }^{34}$.

31 Según el artículo 3.10 de la directiva, el «permiso de almacenamiento" es la "decisión o decisiones escritas y razonadas por las que se autoriza el almacenamiento geológico de $\mathrm{CO}_{2}$ en un emplazamiento de almacenamiento por parte del titular y se especifican las condiciones en las que debe realizarse, expedidas por la autoridad competente de conformidad con las disposiciones de la presente directiva".

32 LAGUNA DE PAZ, José Carlos. La autorización administrativa. Madrid: Thomson Civitas, 2007, pp. 83ss. 33 Ver considerandos 19 y 22.

34 SANZ RUBIALES, Íñigo. «El almacenamiento geológico de dióxido de carbono en la directiva 2009/31, de 23 de abril», pp. 81-82.

LA CAPTURA Y

ALMACENAMIENTO

DE CARBONO:

UNA NOVEDOSA

TÉCNICA DE

REDUCCIÓN

DE EMISIONES DE

GASES DE EFECTO

INVERNADERO

REGULADA

POR LA UNIÓN

EUROPEA

CARBON CAPTURE

AND STORAGE:

A NOVEL

TECHNIQUE

FOR REDUCING

GREENHOUSE

GAS EMISSIONS

REGULATED BY

THE EUROPEAN

UNION 


\section{II.3. El carácter marginal de la captura y el transporte de $\mathrm{CO}_{2}$ en la regulación}

De las diversas fases del proceso vinculado a la tecnología CAC - captura, transporte y almacenamiento— ${ }^{35}$, la directiva dedica casi todo su esfuerzo regulador al almacenamiento. Deja de lado, en bastante medida, la captura y el transporte de dióxido de carbono ${ }^{36}$.

\section{II.3.1. Captura: limitaciones ambientales}

Los escasos preceptos que la directiva dedica específicamente a la captura son, realmente, modificaciones de otras directivas, especialmente de la relativa a la Prevención y Control Integrados de la Contaminación que «permite regular [...] los riesgos de la captura de $\mathrm{CO}_{2}$ para el medio ambiente y la salud humana y por consiguiente debe aplicarse a la captura de flujos de $\mathrm{CO}_{2}$ " (considerando 16 y artículo 37). También la directiva EIA (Environmental Impact Assessment) se aplica a los proyectos de captura y transporte (artículo 31 directiva 2009/31).

\section{II.3.2. Transporte: el acceso de terceros a la red}

En la medida en que la mayor parte de los emisores se sitúan en áreas industriales y los lugares de almacenamiento suelen estar en zonas alejadas de la población, el proceso de CAC exige el transporte del $\mathrm{CO}_{2}$ capturado. Implícitamente, la directiva se refiere al transporte por tuberías subterráneas, suficientemente seguro y que permite crear una red ${ }^{37}$. El artículo 21, relativo al «acceso a la red de transporte y a los emplazamientos de almacenamiento», impone a los Estados la obligación de garantizar el acceso de terceros a las redes y a los emplazamientos de almacenamiento. Esta técnica (Third Party Access, TPA) ha venido siendo aplicada ya por la normativa comunitaria en materia de energía (electricidad o gas ${ }^{38}$ ). No cuestiona ni impone la titularidad pública o privada de dichas instalaciones. Simplemente afirma el derecho de acceso de todos a ellas, incluso en el caso de que sean privadas. Habrá que estar atentos, en este caso, a lo que especifique el ordenamiento

35 BANKES, Nigel \& Martha ROGGENKAMP. «Legal Aspects of Carbon Capture and Storage». En AUTORES VARIOS. Beyond the Carbon Economy. Energy Law in Transition. Oxford: Oxford University Press, 2008, pp. 345-348.

36 Como señala Gámez Mejías, de las tres fases del proceso, la relativa al almacenamiento era la más necesitada de una regulación jurídica específica, según los informes que manejó la Comisión (entre ellos, el de la IPCC). Por eso la directiva se refiere sobre todo a ella (ob. cit., p. 51). La ley española 40/2010 mantiene el mismo planteamiento (ver ALENZA GARCíA, José Francisco. Ob. cit., pp. 304-305).

37 RogGenKAMP, Martha \& Avelien HAAN-KAMmINGA. " $\mathrm{CO}_{2}$ Transportation in the European Union: Can the Regulation of $\mathrm{CO} 2$ Pipelines Benefit from the Experiences of the Energy Sector?». En AUTORES VARIOS. Carbon Capture and Storage. Emerging Legal and Regulatory Issues, pp. 107-108.

38 Directiva 2003/54/CE, de 26 de junio de 2003 sobre normas comunes para el mercado interior de la electricidad (artículo 20); directiva 2003/55/CE de 26 de junio de 2003 sobre normas comunes para el mercado interior del gas natural (artículos 18 y 19); (ver ROGGENKAMP, Martha. «The Concept of Third Party Access Applied to CCS». En RogGenKAMP, Martha \& Edwin WoERDMAN (Eds.). Legal Design of Carbon Capture and Storage. Developments in the Netherlands form an International and EU Perspective. Groningen: Univeristy of Groningen, pp. 280ss.). En el ordenamiento español puede verse, por ejemplo, SUÁREZ SARO, Joaquín. "El acceso de terceros a las redes de gas y electricidad». En SANTOS RECH, Miriam \& Fernando CAMEO BEL (coords.). Derecho de la energía. Madrid: La Ley, 2006, pp. 577ss. 
jurídico interno, que será el que determine la normativa aplicable a la red (y en su caso, al emplazamiento) y las condiciones de construcción y utilización de ambos.

Rigen, así, los mismos principios que para las redes de abastecimiento energético, de acuerdo con lo que viene estableciendo el derecho comunitario en materia de servicios abiertos a la competencia, por estrictas razones de eficiencia. De hecho, como afirma la directiva (considerando 38), «el acceso a las redes de transporte y a los emplazamiento de $\mathrm{CO}_{2}$, con independencia de la ubicación geográfica de los usuarios potenciales en la Unión podría condicionar la entrada o la competitividad en el mercado interior de la electricidad y de la energía». Se impone, por lo tanto, el acceso abierto a todos, de forma transparente y no discriminatoria, para no distorsionar el mercado interior de la electricidad.

El titular de la red (o del permiso de almacenamiento) únicamente podrá denegar el acceso de terceros por razones de falta de capacidad y de forma motivada. Pero esa misma causa de denegación se configura como supuesto de hecho para la realización de las mejoras necesarias en la red o en el emplazamiento para posibilitar precisamente ese acceso, siempre que sea económicamente viable (a cargo de los potenciales clientes) y que no implique daños al medio ambiente.

Ahora bien, las tuberías de $\mathrm{CO}_{2}$ vienen a ser como oleoductos en sentido contrario, que en vez de sacar petróleo del subsuelo, introducen en él dióxido de carbono. Por eso el régimen de los oleoductos o gasoductos puede ser un punto de referencia, no solo para el acceso de terceros a la red, sino también para establecer el régimen de seguridad, de forma que se eviten duplicaciones normativas innecesarias ${ }^{39}$.

Finalmente, los eventuales conflictos que puedan surgir en relación con el acceso a la red de transporte y a los emplazamientos deberán resolverse por mecanismos de solución de conflictos instaurados por los Estados miembros al transponer la directiva, mecanismos entre los que se debe incluir una autoridad independiente de las partes con acceso a toda la información pertinente (artículo 22.1).

\section{TÉCNICAS ECONÓMICAS. ELPRINCIPIO «QUIEN CONTAMINA, PAGA»: RESPONSABILIDAD Y RÉGIMEN DE GARANTÍAS}

\section{III.1. Responsabilidad ambiental}

La responsabilidad de los gestores de estas instalaciones no solo hace referencia (muy lejana) a la eventual incidencia en el cambio climático,

LA CAPTURA Y ALMACENAMIENTO DE CARBONO: UNA NOVEDOSA TÉCNICA DE REDUCCIÓN DE EMISIONES DE GASES DE EFECTO INVERNADERO

REGULADA POR LA UNIÓN EUROPEA

CARBON CAPTURE AND STORAGE: A NOVEL

TECHNIQUE FOR REDUCING GREENHOUSE GAS EMISSIONS REGULATED BY THE EUROPEAN UNION 
sino también al influjo en el medio ambiente («responsabilidad medioambiental»). Esta responsabilidad está regulada por la directiva y en buena medida remitida en su regulación a algunas Directivas ambientales modificadas por la directiva «CAC». La finalidad de estas modificaciones es, pues, que la realización de actividades y la instalación de infraestructuras destinadas a impedir la emisión de gases de efecto invernadero (esto es, un objetivo ambiental claro) no implique a su vez daños al medio ambiente y también, en su caso, a la salud. Estas modificaciones son las siguientes:

- Sujeción de las instalaciones de captura, transporte y almacenamiento de dióxido de carbono a evaluación ambiental. La directiva 2009/31 añade varios supuestos a los listados de proyectos sometidos a evaluación de impacto ambiental; en el anexo I se añaden las instalaciones de captura (si se superan las 1,5 megatoneladas anuales), las de transporte (si superan los ochocientos milímetros de diámetro y los cuarenta kilómetros de longitud), y los emplazamientos de almacenamiento regulados por la directiva 2009/31. En el anexo II (esto es, en el listado de proyectos sometidos a preevaluación o a un régimen de umbrales) se incluyen ahora las instalaciones de captura y transporte de $\mathrm{CO}_{2}$ no incluidos en el anexo I.

- Inclusión de la explotación de almacenes de $\mathrm{CO}_{2}$ entre las actividades generadoras de responsabilidad objetiva por daños ambientales. La directiva «secuestro» añade la explotación de estos almacenes de dióxido de carbono al listado del anexo III de la directiva 2004/35 sobre responsabilidad ambiental, de tal forma que los eventuales daños ambientales producidos por la gestión de estas instalaciones se someten al régimen de responsabilidad objetiva a las instalaciones previstas en el anexo III.

- Inclusión, igualmente, de las instalaciones de captura de dióxido de carbono entre las sometidas a la autorización integrada ambiental, regulada por la directiva 2008/1, relativa a la prevención y control integrados de la contaminación.

Es conocido que el dióxido de carbono no es, en condiciones normales, tóxico, sin perjuicio de la eventual incidencia en el cambio climático o de la posibilidad de encontrar fuertes concentraciones en determinados momentos o situaciones, derivadas normalmente de fugas o de una combustión intensiva. Pero al margen de estas posibilidades, el flujo de $\mathrm{CO}_{2}$ «capturado», transportado y almacenado puede contener trazas o elementos de otros compuestos tóxicos, en mayor o menor magnitud. Y es en esos elementos ajenos al dióxido de carbono donde se puede encontrar la causa de riesgos ambientales. Por eso la directiva exige el aislamiento del $\mathrm{CO}_{2}$ y responsabiliza de los efectos de la operación a los 
titulares de las instalaciones, como recuerda el Preámbulo de la directiva CAC (27):

[...] es necesario imponer limitaciones relativas a la composición del flujo de $\mathrm{CO}_{2}$ coherentes con el propósito principal del almacenamiento geológico, consistente en aislar las emisiones $\mathrm{de}_{2}$ de la atmósfera, y que estas limitaciones estén dictadas por los riesgos que puede suponer la contaminación para la fiabilidad y seguridad de la red de transporte y almacenamiento y para el medio ambiente y la salud humana. Para ello, conviene comprobar la composición del flujo de $\mathrm{CO}_{2}$ previamente a su inyección y almacenamiento.

Estos controles del flujo - sigue explicando la directiva — deben llevarse a cabo en el marco de las Directivas reformadas de Impacto Ambiental y de Prevención y control Integrado de la Contaminación que incluyen las instalaciones de captura en su regulación, y de la propia directiva 2009/31, en virtud de la cual:

[...] el titular del emplazamiento de almacenamiento solo debe aceptar e inyectar flujos de $\mathrm{CO}_{2}$ si se ha llevado a cabo un análisis de la composición, incluidas las sustancias corrosivas, de los flujos y una evaluación de riesgos y si esta ha mostrado que los niveles de contaminación de los flujos de $\mathrm{CO}_{2}$ son conformes a los criterios de composición contemplados en la presente directiva.

En concreto, la presencia de cantidades considerables de impurezas en el $\mathrm{CO}_{2}$ transportado puede incrementar los eventuales impactos potenciales de una fuga o de una ruptura de la tubería de transporte (IPCC, 2005). Entre estas impurezas pueden encontrarse sustancias tóxicas, como el $\mathrm{CO}, \mathrm{NO}_{2}, \mathrm{SO}_{2} \mathrm{y} \mathrm{H}_{2} \mathrm{~S}$, por lo que es importante controlar su concentración ${ }^{40}$.

También, lógicamente, hay que asegurar la estanqueidad de los almacenes subterráneos, para evitar daños ambientales o de carácter sanitario en el caso de que los gases almacenados puedan incluir, en mayor o menor medida, un cierto componente de dichas sustancias tóxicas ${ }^{41}$.

40 European Commission. Implementation of Directive 2009/31/EC on the Geological Storage of Carbon Dioxide. Guidance Document 2. Characterisation of the Storage Complex, $\mathrm{CO}_{2}$ Stream Composition, Monitoring and Corrective Measures. Bruselas, 2011, p. 77.

41 Ver, a este respecto, el recurso por omisión (artículo 265 del Tratado de Funcionamiento de la Unión Europea) interpuesto ante el Tribunal de Justicia de la UE el 16 de abril de 2007 - Scientific and Technological Committee y otros/Potocnik y otros, miembros de la Comisión (Asunto T-125/07), porque se basa en los riesgos medioambientales y para la salud pública que pueden derivar de la actividad de almacenamiento de dióxido de carbono. En él, los demandantes - Scientific and Technological Committee of AGH University of Science and Technology y otros (Cracovia, República de Polonia) - solicitaban que se declarase que varios miembros de la Comisión Europea habían incurrido en una omisión al no haber adoptado medidas adecuadas algunas para prevenir las consecuencias negativas de la aplicación de las técnicas de almacenamiento de $\mathrm{CO}_{2}$ sin recipiente en depósitos geológicos, tras haber sido informados por parte de los demandantes de los riesgos

LA CAPTURA Y ALMACENAMIENTO DE CARBONO: UNA NOVEDOSA TÉCNICA DE REDUCCIÓN DE EMISIONES DE GASES DE EFECTO INVERNADERO

REGULADA

POR LA UNIÓN EUROPEA

CARBON CAPTURE AND STORAGE:

A NOVEL

TECHNIQUE FOR REDUCING GREENHOUSE GAS EMISSIONS REGULATED BY THE EUROPEAN UNION 


\section{III.2. «Responsabilidad por daños climáticos»42}

La directiva «secuestro» (2009/31) menciona en diversos momentos la denominada «responsabilidad por daños climáticos» que se atribuye a los titulares de instalaciones CAC y que se concreta en la obligación de entregar derechos de emisión en caso de fugas. No deja de sorprender que se califique como «responsabilidad por daños climáticos» cuando la aportación al cambio climático de una determinada fuga de dióxido de carbono de una instalación es prácticamente inapreciable ${ }^{43}$. Pero —es cierto- tampoco es una responsabilidad exclusivamente ambiental ${ }^{44}$. De ahí el cambio de título de imputación, que permite acrecentar notablemente la exigencia económica. En concreto, el considerando 30 recuerda:

Conviene establecer disposiciones relativas a la responsabilidad por daños causados al medio ambiente local y por daños al clima resultantes de un fallo del confinamiento permanente de $\mathrm{CO}_{2}[\ldots]$. La responsabilidad por daños al clima debido a fugas está cubierta por la inclusión de los emplazamientos de almacenamiento en la directiva 2003/87/CE, que requiere la entrega de derechos de emisión en caso de fugas.

Y en esa línea, los artículos $11.4,17.2,17.4$ y 18.1 de la misma directiva 2009/31 aluden igualmente a la necesidad de entrega de derechos del ETS (European Trading System)

en el caso de que se produzcan fugas en el almacenamiento del dióxido de carbono.

Por lo tanto, es la directiva 2009/29, de reforma del mercado de emisiones, la que regula lo que se ha dado en llamar «responsabilidad por daños climáticos» de los titulares de instalaciones CAC. ¿Cómo opera dicha responsabilidad? Básicamente opera a través de la inclusión de las instalaciones CAC en el ámbito subjetivo de aplicación de la

que para la salud de las personas y para el medio ambiente se derivan de tales técnicas. Los demandantes alegaban que los miembros de la Comisión demandados no se pronunciaron de ningún modo sobre los problemas expuestos en las denuncias que se les presentaron y en las que se les solicitaba que adoptaran medidas adecuadas y llevaran a cabo los estudios propuestos por los demandantes sobre los efectos del almacenamiento de dióxido de carbono a escasa profundidad. En su opinión, esta omisión infringía el ordenamiento jurídico dde la Unión Europea y era incompatible con las obligaciones de un miembro de la Comisión. El recurso, no obstante, fue inadmitido por Auto del Tribunal de Primera Instancia de 25 de marzo de 2009, que además condenó en costas a los demandantes (DOUE C-129, de 6 de junio de 2009, pp. 13-14).

42 Ver, in extenso, SANz RuBIALES, Íñigo. «El almacenamiento geológico de dióxido de carbono en la directiva 2009/31, de 23 de abril», pp. 93-98.

43 Ver DOMÉNECH PASCUAL, Gabriel. «¿Es proporcionado cerrar una empresa por infracciones a la Ley del mercado de derechos de emisión de gases de efecto invernadero?». Revista Aranzadi de Derecho Ambiental, 9 (2006), pp. 83-85.

44 Sobre los diversos regímenes de responsabilidad que pueden vincularse a los daños derivados del cambio climático, ver TORRE-SCHAUB, Marta. «La gouvernance du climat: vieilles notions pour nouveaux enjeux». Cahiers Droit, Sciences \& Technologies, 2 (2009), Dossier Thématique Droit et Climat, pp. 143ss. 
directiva «mercado». A partir de 2013, las instalaciones CAC, que hasta el momento quedaban al margen de la directiva «mercado» 2003/87, pasarán a someterse a esta, tal y como establece en su anexo $\mathrm{I}^{45}$.

Ahora bien, dichas instalaciones no serán acreedoras de la asignación gratuita de derechos de emisión, tal y como establece el artículo 10 bis.3 de la misma directiva ${ }^{46}$. Por tanto, las emisiones producidas por las fugas, constatadas y validadas, deberán ser respaldadas por derechos de emisión. Derechos que, por otra parte, deberán ser adquiridos en el mercado o (parcialmente) mediante la realización de proyectos generadores de créditos de carbono, porque los titulares de instalaciones CAC no pueden obtener cuotas gratuitas. Se trata, así, de una responsabilidad patrimonial, diversa de la estrictamente ambiental en la medida en que se basa en la presunción de la incidencia que las emisiones de $\mathrm{CO}_{2}$ «fugadas» pueden producir en el clima, y que se satisface mediante la entrega de títulos con valor económico, como son las cuotas o derechos de emisión (aunque no cabe satisfacer dicha responsabilidad mediante el equivalente pecuniario de los derechos correspondientes a las toneladas «emitidas» por fuga: la «moneda de pago» es únicamente los derechos de emisión).

En todo caso, la inclusión de las instalaciones CAC en el ámbito subjetivo de aplicación de la directiva mercado tiene una serie de consecuencias que hay que tener en cuenta.

Por una parte, las instalaciones CAC tienen que estar incluidas (por sí solas, o como instalaciones complementarias de una instalación ya sometida a la directiva) en la correspondiente autorización de emisiones, tal y como establece la Decisión de la Comisión de 8 de junio de 2010, por la que se modifica la Decisión 2007/589 a fin de incluir directrices para el seguimiento y la notificación de emisiones de gases de efecto invernadero derivadas de la captura, el transporte y el almacenamiento geológico de dióxido de carbono (DOUE del 22) ${ }^{47}$.

45 Ver el nuevo anexo I (Categorías de actividades a las que se aplica la presente directiva ), que deroga al anterior y que incluye: «captura de gases de efecto invernadero de las instalaciones cubiertas por la presente directiva con fines de transporte y almacenamiento geológico en un emplazamiento de almacenamiento autorizado de conformidad con la directiva 2009/31/CE»; «transporte de gases de efecto invernadero a través de gasoductos confines de almacenamiento geológico en un emplazamiento de almacenamiento autorizado de conformidad con la directiva 2009/31/CE»; «almacenamiento geológico de gases de efecto invernadero en un emplazamiento de almacenamiento autorizado de conformidad con la directiva 2009/31/CE».

46 «Sin perjuicio de los apartados 4 y 8 y no obstante lo dispuesto en el artículo 10 quáter, no se asignará ningún derecho de forma gratuita a los generadores de electricidad, a las instalaciones de captura de $\mathrm{CO}_{2}$, a las conducciones para el transporte de $\mathrm{CO}_{2}$ ni a los emplazamientos de almacenamiento de $\mathrm{CO}_{2}$ ".

47 Nuevo anexo XVI: «La captura de $\mathrm{CO}_{2}$ puede ser realizada bien por instalaciones especializadas que reciban $\mathrm{CO}_{2}$ por transferencia de otras instalaciones o bien por instalaciones que desempeñen actividades emisoras de $\mathrm{CO}_{2}$ que deba ser capturado en virtud de la misma autorización de emisión de gases de efecto invernadero. Todas las partes de la instalación dedicadas a la captura de $\mathrm{CO}_{2}$, al almacenamiento intermedio, a la transferencia a una red de transporte de $\mathrm{CO}_{2} \mathrm{O}$ a un emplazamiento para almacenamiento geológico de emisiones de $\mathrm{CO}_{2}$ deben estar incluidas en la autorización de emisión de gases de efecto invernadero. En caso de que la instalación realice otras actividades

LA CAPTURA Y

ALMACENAMIENTO

DE CARBONO:

UNA NOVEDOSA

TÉCNICA DE

REDUCCIÓN

DE EMISIONES DE

GASES DE EFECTO

INVERNADERO

REGULADA

POR LA UNIÓN

EUROPEA

CARBON CAPTURE

AND STORAGE:

A NOVEL

TECHNIQUE

FOR REDUCING

GREENHOUSE

GAS EMISSIONS

REGULATED BY

THE EUROPEAN

UNION 
Por otra parte, estas instalaciones pueden ser las únicas que deben respaldar a título principal emisiones no voluntarias, o emisiones no derivadas inmediatamente de una actividad de quema de combustibles fósiles, sino de fugas en la captura, transporte o inyección de dióxido de carbono. Tanto es así que una instalación CAC que funcione correctamente podría tener emisiones tan reducidas que, en condiciones normales, no exigirían posiblemente la inclusión de dicha actividad en el ámbito de aplicación de la directiva. La citada Decisión de 8 de junio de 2010 alude en sus anexos XVI a XVIII a las posibles fuentes de emisión de $\mathrm{CO}_{2}$ y además de las fugas incluye la posible utilización de combustibles fósiles para el funcionamiento de la instalación en cuestión (estaciones de compresión, centrales eléctricas in situ, por ejemplo) así como la ventilación de la red de transporte o en el momento de la inyección, emisiones todas ellas cuantitativamente reducidas en relación con el volumen de $\mathrm{CO}_{2}$ capturado, transportado o inyectado.

Así se confirma que la inclusión de estas instalaciones en el ámbito subjetivo de aplicación de la directiva «mercado» cumple únicamente la función de imponer la obligación de respaldar las eventuales fugas que puedan producirse en el proceso CAC con derechos de emisión y no la de estimular este tipo de instalaciones, como se puede confirmar a continuación.

El artículo 12 bis.3 de la directiva 2009/29 establece que

No habrá obligación de entregar derechos de emisión relativos a emisiones cuya captura esté comprobada y que se hayan transportado para su almacenamiento permanente a una instalación con un permiso vigente de conformidad con la directiva 2009/31/CE del Parlamento Europeo y del Consejo, de 23 de abril de 2009, relativa al almacenamiento geológico de dióxido de carbono.

Lo no-emitido, lógicamente, son derechos ahorrados. Pero no corresponden a derechos gratuitamente recibidos y no utilizados, porque como consecuencia del sometimiento de las instalaciones CAC a la directiva mercado desde 2013, y de la inaplicación de la asignación gratuita a este tipo de instalaciones, no tienen derecho a la asignación gratuita de dichas cuotas. Como dice la directiva en su considerando 20: «El principal incentivo a largo plazo para las actividades de captura y almacenamiento de carbono y las nuevas tecnologías de energías renovables consiste en que no será necesario entregar los derechos de 
emisión por las emisiones de $\mathrm{CO}_{2}$ que se almacenen permanentemente o se eviten» ${ }^{48}$.

\section{III.3.Garantías financieras y transferencia de responsabilidad ${ }^{49}$}

Una vez que ha obtenido el «permiso» de almacenamiento, el titular de la actividad puede llevar a cabo la inyección de dióxido de carbono en la formación geológica. A él le corresponde el seguimiento de las instalaciones de inyección y de almacenamiento, de acuerdo con el artículo $13^{50}$, y el ejercicio de la obligación de informar a la autoridad competente en diversas situaciones, y todo ello sin perjuicio de la potestad inspectora administrativa (artículo 15). El titular asume la responsabilidad ambiental derivada del funcionamiento de las instalaciones (de acuerdo con las previsiones de la directiva 2004/35 y concordantes) y la llamada «responsabilidad por daños al clima» (ver supra), de acuerdo con la directiva 2003/87 y su modificación ${ }^{51}$.

La directiva articula dos tipos de garantías financieras que corren a cargo del titular de la instalación y que cubren diversos ámbitos de responsabilidad de este a lo largo del funcionamiento y del periodo posterior a la clausura del almacén subterráneo ${ }^{52}$. Por una lado tenemos la «garantía financiera» propiamente dicha (artículo 19). En la solicitud del permiso de almacenamiento se debe presentar la prueba de la constitución de esta garantía financiera (artículo 7.10). Y debe adaptarse periódicamente a la luz de los cambios en el riesgo de fuga y de los precios de los derechos de emisión.

Cubre, por una parte, los riesgos de fuga y el cumplimiento de las obligaciones impuestas por el permiso o por la autoridad competente durante el desarrollo de la actividad de almacenamiento. En el caso de cierre del emplazamiento, cubrirá las obligaciones del titular hasta la transferencia de responsabilidad a la autoridad competente; y, en el caso

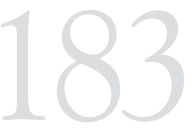

LA CAPTURA Y ALMACENAMIENTO DE CARBONO:

UNA NOVEDOSA

TÉCNICA DE

REDUCCIÓN

DE EMISIONES DE

GASES DE EFECTO

INVERNADERO

REGULADA

POR LA UNIÓN

EUROPEA

CARBON CAPTURE

AND STORAGE:

A NOVEL

TECHNIQUE

FOR REDUCING

GREENHOUSE

GAS EMISSIONS

REGULATED BY

THE EUROPEAN

UNION

48 Efectivamente, a corto plazo, el bajo precio del carbono y el hecho de que la tecnología CAC esté todavía «en mantillas» hace que no sea viable económicamente la introducción de este tipo de instalaciones; por eso es preciso incentivar de diversos modos su construcción. Sin embargo, a medio o largo plazo, el precio del carbono y la accesibilidad de los avances tecnológicos presumiblemente harán que dicha tecnología sea rentable (ver NORDHAUS, Robert R. «CCS Under GHG Regulatory Programmes». En AUTORES VARIOS. Carbon Capture and Storage. Emerging Legal and Regulatory Issues, p. 84).

49 Sobre las cuestiones relativas a la transferencia de responsabilidad, ver European Commission. Implementation of Directive 2009/31/EC on the Geological Storage of Carbon Dioxide. Guidance Document 3. Criteria for Transfer of Responsibility to the Competent Authority. Bruselas, 2011.

50 El seguimiento debe ir acompañado de la presentación —al menos anual— de los correspondientes informes dirigidos a la autoridad pública competente sobre el funcionamiento de las instalaciones (artículo 14) y de la notificación de fugas o irregularidades significativas, cuando se produzcan (artículo 16.1).

51 Ver considerando 30; artículos 17.2, 18.1, 34

52 Sobre las garantías señaladas, las opciones de regulación posibles para los Estados miembros y las sugerencias de la Comisión sobre los tipos de garantías, ver European Commission. Implementation of Directive 2009/31/EC on the Geological Storage of Carbon Dioxide. Guidance Document 4. Article 19 Financial Security and Article 20 Financial Mechanism. Bruselas, 2011. 
de retirada del permiso, debe servir para financiar el funcionamiento de la instalación hasta la expedición de un nuevo permiso (artículo 19.3) ${ }^{53}$. Pero este planteamiento «expansivo» del objeto garantizado (todas las obligaciones) encarece excesivamente el coste de esta garantía y exige que la aplicación de esta previsión se haga con realismo ${ }^{54}$.

El problema de esta garantía es que, a la luz de los términos de la directiva, no cubre más que la responsabilidad «por cambio climático» y deja fuera la responsabilidad «medioambiental»: se deja a los Estados miembros la elección de los instrumentos financieros que puedan cubrir esta responsabilidad 5 .

El «mecanismo financiero» del artículo 20, el llamado «mecanismo financiero», es otra garantía a la que se le ha cambiado el nombre dejando el apellido, y cuya existencia autónoma no resulta suficientemente justificada a la luz de la redacción de la directiva. De hecho, no se preveía en la propuesta de directiva y se añadió - cambiando así el criterio original de asunción de los costes tras la transferencia de la responsabilidad — durante la tramitación de esta, lo cual puede justificar la falta de estudio suficiente de su ubicación sistemática.

El artículo 20 se refiere a una «contribución financiera» que se hace a la autoridad competente antes de que se produzca la transferencia de responsabilidad en situaciones normales y que se destina a cubrir el seguimiento de la instalación y, en su caso, los costes necesarios para garantizar el confinamiento completo y permanente del $\mathrm{CO}_{2}$ tras la transferencia y por un periodo de treinta años. Aunque su objetivo es aparentemente modesto, lo cierto es que a largo plazo puede ser muy eficaz para cubrir las contingencias posteriores a la transferencia ${ }^{56}$.

El objeto, en ambos casos (mecanismo y garantía) es parcialmente el mismo - cubrir los costes de supervisión-, pero aplicado en momentos diversos. Por eso, la principal diferencia con la garantía stricto sensu es, más que el objeto, el momento de su constitución. Mientras que esta debe constituirse antes de la solicitud del permiso, la contribución del «mecanismo» debe hacerse antes de la transferencia de responsabilidad.

53 «La directiva, al establecer la necesidad de una garantía financiera que cubra la responsabilidad previa a iniciar la inyección [artículo 19] se ve obligada a determinar que la misma se adaptará periódicamente a los cambios sobre la evaluación de riesgos [...]. Esto conduce al extremo de no poder ni siquiera establecer unos porcentajes mínimos o máximos para la misma por la sencilla razón de que en el presente no hay datos que justifiquen una u otra cantidad, cualquier determinación sería absolutamente aleatoria» (GÁmEZ MEJíAS, Manuel. Ob. cit., p. 61).

$54 \mathrm{La}$ versión inglesa - no la española - establece que esta garantía se crea «to ensure that all obligations [...] can be met" (ver CLARKE, Chris. "Long-Term Liability for CCS: Some Thoughts about Specific Risks, Multiple Regimes and the EU Directive». En AUTORES VARIOS. Carbon Capture and Storage. Emerging Legal and Regulatory Issues, p. 196.

55 L. cit.

56 Ver ibídem, p. 197 
En resumen, tras el cierre autorizado de un lugar de seguimiento, el titular de la concesión será el responsable jurídico y económico del seguimiento de dicho lugar durante un plazo mínimo de veinte años. Esa responsabilidad económica estará cubierta por la «garantía financiera» que debe constituirse antes de que comience la inyección del carbono (artículo 19). Y cuando hayan transcurrido esos veinte años del cierre - y se cumplan las otras condiciones establecidas — la responsabilidad del seguimiento se transfiere a la Administración. Pero la responsabilidad financiera seguirá siendo del titular de la concesión durante al menos otros treinta años y se hará efectiva a través de las aportaciones económicas que anticipadamente debe realizar al «instrumento financiero» (artículo 20) previsto al efecto ${ }^{57}$.

Como se ha dicho antes, el objetivo del almacenamiento geológico del dióxido de carbono es su confinamiento permanente. Por esa razón, la directiva impone al titular de la instalación el seguimiento del funcionamiento de esta con posterioridad al cierre, durante al menos veinte años. Pero, lógicamente, esta responsabilidad no puede extenderse sine $\mathrm{di}^{58}$. Por eso la directiva prevé la transferencia de las obligaciones de seguimiento y, en su caso, medidas correctoras, preventivas, reparadoras y de entrega de derechos de emisión ${ }^{59}$ (artículos 17-18).

Ahora bien, aunque la transferencia conlleva, en principio, que las responsabilidades reguladas por la directiva «secuestro» (2009/31), por la directiva «responsabilidad» $(2004 / 35)$ y por la directiva «mercado» (2003/87) pasan a la autoridad competente del Estado correspondiente, lo cierto es que puede ocurrir que no se transfiera toda la responsabilidad y que el operador de la instalación siga siendo responsable en algunos ámbitos, por causas diversas: por la dificultad para conseguir una evidencia del completo y permanente confinamiento del dióxido de carbono; por el hecho de que la responsabilidad de derecho civil no se transfiere y la normativa estatal puede atribuir la responsabilidad al antiguo titular (por ejemplo, en materia de suelos contaminados, calidad de las aguas, salud pública, etcétera); además, en algunos casos se puede posponer la transferencia por problemas pendientes, todavía no resueltos. Todo ello, sin perjuicio de la potestad de la Administración de recuperar los costes realizados en los casos en los que el titular haya incurrido en falta, en particular en los casos de datos deficientes, ocultación de información

57 AlenZa García, José Francisco. Ob. cit., p. 321.

58 Frente a este planteamiento, europeo, de transferencia a largo plazo de la responsabilidad a los Estados miembros, algunos autores han entendido que el principio "quien contamina paga» exigiría el mantenimiento de la responsabilidad en manos de los titulares (ver BAILEY, Paul \& otros. «Can Governments Ensure Adherence to the Polluter Pays Principle in the Long-Term CCS Liability Context?». Sustainable Development Law \& Policy, XII, 2 (2012), pp. 46-70, pp. 48, 51ss). El problema de esta solución está en que difícilmente puede exigirse al particular que asuma la responsabilidad patrimonial si es incierta y a larguísimo plazo.

59 AlenZa García, José Francisco. Ob. cit., p. 320.

LA CAPTURA Y

ALMACENAMIENTO

DE CARBONO:

UNA NOVEDOSA

TÉCNICA DE

REDUCCIÓN

DE EMISIONES DE

GASES DE EFECTO

INVERNADERO

REGULADA

POR LA UNIÓN

EUROPEA

CARBON CAPTURE

AND STORAGE:

A NOVEL

TECHNIQUE

FOR REDUCING

GREENHOUSE

GAS EMISSIONS

REGULATED BY

THE EUROPEAN

UNION 
pertinente, negligencia, engaño intencionado o ausencia de la diligencia debida (artículo 18.7) ${ }^{60}$.

\section{RECAPITULACIÓN}

La directiva 2009/31, de 23 de abril, transpuesta en España por la ley 40/2010, de 29 de diciembre, regula el almacenamiento geológico del dióxido de carbono emanado de grandes instalaciones de combustión.

Se trata de una técnica de «transición», necesaria a medio plazo para evitar las emisiones de dióxido de carbono a la atmósfera emanadas de las (todavía) imprescindibles centrales térmicas, hasta que las energías renovables estén totalmente desplegadas. Regula fundamentalmente el almacenamiento, y con carácter complementario la captura y el transporte de estos gases de efecto invernadero.

La directiva no establece la obligación de construir este tipo de instalaciones, aunque estimula la implantación de esta tecnología y admite la posibilidad de convertirla en obligatoria a partir de 2015. Los Estados pueden, discrecionalmente, permitir o no este tipo de instalaciones en su territorio, pero si lo hacen deben someterse a los dictados de la directiva y exigir, por una parte, los correspondientes permisos de exploración (en su caso) y de almacenamiento (auténticas concesiones en el caso español); por otra, la responsabilidad ambiental y por cambio climático de los titulares —incluso después del cierre-; y, finalmente, asumir la transferencia de responsabilidad cuando se den los requisitos exigidos por la directiva, tras el transcurso de —al menosveinte años. 